$10.2478 / \mathrm{gb}-2021-0013$

sciendo

\title{
Einige Bemerkungen zu den produktivsten Wortbildungsstrategien in der deutschen Fachterminologie des Eisenbahnwesens unter der kontrastiven Berücksichtigung des Polnischen

\author{
Sebastian MAŚLANKA \\ M.A.; Universität Opole; \\ E-Mail: sebastian.maslanka@uni.opole.pl
}

\begin{abstract}
This article is dedicated to present the most common word-formation strategies in German railway specialist terminology. The presentation of the most productive morphological nomination strategies in the analysed area is preceded by a short historical outline of the railway industry in Germany. The adumbration of the historical background and hints regarding the current EU environmental plans for railways are intended to expose the importance and relentless currency of the analysis undertaken here. The presented examples, which were extracted from the most extensive industry terminology database, RaiLLexic, have been correlated with their Polish equivalents. This contrastive procedure allows, going further, to draw conclusions about both language systems within which the professional terminology described here is constituted.
\end{abstract}

Key words: language for a special purpose, railroad industry, examination of professional languages, terminology

\section{Die Eisenbahn als Träger der nationalen Identität}

Ein Rückblick auf die Geschichte Deutschlands offenbart ein beinahe reziprokes Verhältnis zwischen der aufkeimenden Staatlichkeit und dem allmählichen Aufkommen der Eisenbahn. 
Die Rolle des Eisenbahnwesens kann in diesem Kontext auf zweierlei Weise gedeutet werden. Einerseits lässt es sich nämlich als eine Art des besonderen Seismografen betrachten, der die wichtigsten historischen Umwälzungen reflektierte und unter ihrem Einfluss sein Betätigungsfeld laufend anpassen musste. Auf der anderen Seite wäre es jedoch absolut unbegründet, die geschichtliche Bedeutung der Eisenbahn auf die Rolle eines passiven Objektes zu beschränken, ohne ihren aktiven Beitrag zur Gestaltung der wirtschaftlichen, sozialen und politischen Verhältnisse zu erkennen. Der so angedeutete Dualismus liegt nahe, wenn man auf die Geburtsstunde der deutschen Bahn zurückblickt, wo die äußerst zersplitterte Organisationsstruktur des damals bestehenden ${ }^{1}$ Deutschen Bundes mit 37 Bundesstaaten und vier Freien Städten dazu führte, dass der Streckenbau und die Linienführung bis zu den 60ern Jahren des 19. Jahrhunderts von jedem Staat einzeln und in Bezug auf Interessen des Bundes komplett willkürlich geplant und vollzogen wurden. ${ }^{2}$ Es mag demnach als paradox erscheinen, dass eben die Eisenbahn, die vom administrativen Chaos in der ersten Hälfte des 19. Jahrhunderts direkt betroffen war, von vielen damaligen Intellektuellen als eine enorme Chance für die Vereinigung der Nation empfunden wurde. ${ }^{3} \mathrm{Zu}$ jenen Bahnenthusiasten gehörte unter anderem Goethe, der 1828 in einem Gespräch mit seinem Vertrauten, Johann Peter Eckermann behauptete, dass „ihm nicht bange sei, dass Deutschland nie eins werde; da seine [S.M.] guten Chausseen und künftigen Eisenbahnen schon das ihrige tun werden [S.M.]. ${ }^{" 4}$ Von derselben Überzeugung schien ein paar Jahrzehnte später der erste Reichskanzler des Deutschen Reiches, Otto von Bismarck, getrieben zu sein, als

1 Der Bund existierte von 1815 bis 1866 [S.M.].

2 Vgl. Gall, Lothar/ Pohl, Manfred: Die Eisenbahn in Deutschland von den Anfängen bis zur Gegenwart. München 1999, S. 15.

3 Vgl. ebd.

${ }^{4}$ Eckermann, Johann Peter: Gespräche mit Goethe in den letzten Jahren seines Lebens. Wiesbaden 1975, S. 532. 
er 1873 das Reichsbahnamt ins Leben rief und von da an die Vereinigung der bislang privatisierten Eisenbahnnetze anstrebte. ${ }^{5}$ Obwohl die rasante Entwicklung des Schienenverkehrs eine Aufbruchsstimmung in der deutschen Gesellschaft des Industrialisierungszeitalters hervorrief ${ }^{6}$ und die sozialen, wie auch wirtschaftlichen Verhältnisse wesentlich aufbesserte ${ }^{7}$, blieb dieser dynamisch fortschreitende Transportzweig leider von den tragischen Wirren der militärischen Konflikte nicht verschont. Das Potential des Eisenbahnwesens wurde schon in dem Deutsch-Französischen Krieg in den Jahren 1870-71 den Militärzwecken unterworfen. Nicht anders war es auch im Falle der zwei großen Weltkriege des 20. Jahrhunderts. Während jedoch der Einsatz des Schienenverkehrs im Ersten Weltkrieg der Versorgung und Truppenmobilmachung in einem bisher unbekannten Ausmaß diente, wurden die deutschen Güterzüge in der Zeit des Zweiten Weltkriegs indirekt zum Symbol der Ausrottung, da sie die Juden und andere von NSDeutschland verfolgte Gruppen unter menschenunwürdigen

5 Vgl. Lothar Gall/ Manfred Pohl, Die Eisenbahn in Deutschland von den Anfängen bis zur Gegenwart. München 1999, S. 70-80.

${ }^{6}$ Unter denjenigen, die das Aufkommen des neuen Verkehrsträgers euphorisch begrüßten, war unter anderem Heinrich Heine, der in der Augsburger Allgemeinen Zeitung seinen Enthusiasmus mit folgenden Worten ausdrückte: ,Welch Veränderungen müssen jetzt eintreten in unserer Anschauungsweise und in unseren Vorstellungen! Sogar die Elementarbegriffe von Raum und Zeit sind schwanken geworden. Durch die Eisenbahnen wird der Raum getötet und es bleibt uns nur noch die Zeit übrig." (Heine Heinrich: Sämtliche Schriften in zwölf Bänden. Berlin 1981, S. 449)

7 Schon im Jahre 1850 beschäftigten die Bahnen 26000 Personen und galten somit als der größte Arbeitgeber Deutschlands. Diesen Status bewahrte das Bahnwesen bis zum 20. Jahrhundert und es verstärkte seine Relevanz für staatliche Finanzen in jener Zeit zusätzlich dadurch, dass eben die Bahn die meisten Aufträge für Privatunternehmen erteilte. (vgl. Gall Lothar/ Pohl, Manfred: Die Eisenbahn in Deutschland von den Anfängen bis zur Gegenwart. München1999, S. 38-39) 
Bedingungen in die Vernichtungslager transportierten. Die symbolische Präsenz der Bahn manifestiert sich weiterhin in der Nachkriegsgeschichte Deutschlands, wo sie zuerst als ,Trümmerbahn` zur Beseitigung des Schrotts im kriegszerstörten Land eingesetzt wurde, um schließlich selbst dem Schicksal des besiegten Landes zu folgen, wofür man die Spaltung in die Bundesbahn (BRD) und Reichsbahn (DDR) halten kann. ${ }^{8}$ Die Wendezeit schlug sich in der Gründung der Deutschen Bahn AG nieder, die vor der Aufgabe stand, das bislang geteilte Land unter den neuen Bedingungen der kapitalistischen Marktrealität wieder zu vereinen.

\subsection{Die Eisenbahn als Werkzeug für Nachhaltigkeit}

Obwohl die Eisenbahn, wie bereits gezeigt, in der Nationalidentität der Deutschen tief eingewurzelt ist, konnte dieser traditionsreiche Transportzweig im Laufe der letzten Jahrzehnte mit den ,neuen' und oftmals schnelleren Transportmitteln nicht mithalten, was vor allem am gesunkenen Transportvolumen zugunsten des Straßen- und Flugverkehrs abzulesen ist. Die Hoffnung, dieser traditionsreichen Branche ihren alten Glanz wieder zu bringen, wurde bei allen Eisenbahnliebhabern in den letzten Jahren institutionell geweckt. Gemeint ist hier die von der EU befolgte aktuelle Wachstums- und Nachhaltigkeitsstrategie, der sog. Grüne Deal, in dessen Kern die Reduzierung der CO2-Emission um 30 Prozent bis 2033 steht. ${ }^{9}$ Um die ökologische Wende in der Industriegesellschaft der EU zu erreichen, wurde von der EU-Kommission ein Maßnahmenpaket erarbeitet, das unter den vielen Förderformen der nachhaltigen

${ }^{8}$ Die Gründung dieser zwei Gesellschaften war rein administrativ bedingt und glich der Teilung Deutschlands in DDR (Reichsbahn) und BRD (Bundesbahn) [S.M.]

9 Vgl. Die Maßnahmen des Grünen Deals, Zugriff 29.09.2020. https:// ec.europa.eu/info/strategy/priorities-2019-2024/european-greendeal_de (Stand: 30.09.2020) 
Entwicklung u. a. die Belebung des Schienenverkehrs vorsieht. ${ }^{10}$ Die signifikante Rolle der Bahn im Streben nach der Nachhaltigkeit erfährt jüngst eine besondere Hervorhebung, da die Europäische Kommission das Jahr 2021 zum Jahr der Schiene ausrief, um auf diese Weise verstärkt für die Umstellung auf dieses Verkehrsmittel zu werben.

\subsection{Die Interoperabilität im Schienenverkehr}

Will man diese proökologische EU-Strategie in der breiteren Perspektive betrachten, so lassen sich Parallelen zu der Aktivität des Internationalen Eisenbahnverbandes, UIC, erkennen, der seit 1922 seine Anstrengungen unveränderlich darauf ausrichtet, die Interoperabilität im globalen Schienenverkehr zu sichern. ${ }^{11}$ Unter Interoperabilität ist in diesem Zusammenhang die Harmonisierung sämtlicher Eisenbahnnetze zu verstehen, die die grenzübergreifende Nutzung internationaler Eisenbahnstrecken durch alle Züge gewähren sollte. ${ }^{12}$ Die Schaffung der Interoperabilität kann sich dabei nur mehrdimensional vollziehen. Die Reichweite der dazu ergriffenen Maßnahmen erstreckt sich nämlich von der Anpassung der internationalen Bahninfrastruktur $^{13}$ über die Standarisierung des Rechtsrahmens von

${ }^{10}$ Eines der großen Ziele des Europäischen Grünen Deals ist es, drei Viertel aller auf den Straßen beförderten Frachtgüter auf mehr umweltfreundlicheWegezuleiten, wie die Schienen oderBinnenwasserstraßen. Die Maßnahmen des Grünen Deals, Zugriff 29.09.2020 https:// ec.europa.eu/info/strategy/priorities-2019-2024/european-greendeal_de (Stand: 30.09.2020)

${ }^{11}$ Vgl. Ebeling, Klaus: Die Fortentwicklung des Europäischen Eisenbahnsystems - ,Interoperabilität' auch auf sprachlicher Ebene. In: Gerstenkorn, Alfred/ Hums, Lothar/ Schmidt Arnlod (Hgg.): Die Sprache der Bahn. Zur deutschen Eisenbahnsprache im europäischen Kontext. Frankfurt 2006: S. 291-294.

12 Vgl. ebd.

${ }^{13}$ Es handelt sich z. B. um die Angleichung von Spurweiten und Umbau der Tunnels, sodass sie international befahrbar werden. 
Transportvorschriften, bis hin zur Gewährleistung der reibungslosen Verständigung zwischen den Bahnbediensteten aus verschiedenen Ländern.

\section{RailLexic - die umfangreichste Terminologie- datenbank im Dienste der Interoperabilität}

Die essenzielle Bedeutung der Vereinheitlichung von Fachterminologie für die Interoperabilität des Schienenverkehrs wurde bereits im Jahre 1991 erkannt. Einer der Initiatoren dieses Unterfangens war die Deutsche Bahn AG, die eben 1991 in Zusammenarbeit mit dem Internationalen Bahnverband den ersten Terminologieworkshop organisierte. Diese Initiative wurde von anderen europäischen Eisenbahnen sehr positiv aufgenommen und entwickelte sich zu zyklischen internationalen Sitzungen, die den Dolmetschern und Eisenbahnsachverständigen eine Diskussionsebene für terminologische Fragen bot. Die unter dem Namen „RailLexic“ fortgeführten Workshops lieferten 1995 das erste internationale Digitalwörterbuch der Eisenbahnsprache. Während die erste CD-Ausgabe elf Sprachen enthielt, findet man in der aktuellen Version 5.0 Begriffe, die in 23 Sprachen übersetzt wurden. ${ }^{14}$ Die im Folgenden analysierte Terminologiedatenbank ist somit die größte Quelle für den Eisenbahnwortschatz, dervon den internationalen Bahnexperten standarisiert wurde.

\section{Das Belegkorpus und Ziele der Analyse}

Als Belegkorpus für die vorliegende Analyse diente die letzte Ausgabe des besagten Terminologiesystems RailLexic. Aufgrund des gewaltigen Volumens von RailLexic werden im Folgenden nur ausgewählte Phänomene präsentiert, die während

${ }^{14}$ Vgl. UIC, Zugriff 12.12.2019, https://uic.org/support-activities/ terminology/\#RailLexic. 
der Durchleuchtung von $18^{15}$ Themendomänen festgestellt wurden. Der Transparenz halber wird im Folgenden nur solchen Aspekten Rechnung getragen, die aufgrund ihrer überdurchschnittlichen Frequenz als repräsentativ für den ganzen Untersuchungsbereich gelten dürfen. Die Thematik der polnischen Äquivalente, die laut den Informationen von UIC-Terminologen aus dem Deutschen übersetzt wurden, soll dabei in einzelne Segmente fließend integriert werden. Der Fokussierung auf die Terminologie liegt die innerhalb der Fachsprachenforschung oft anzutreffende Überzeugung zugrunde, dass die Lexik das fachliche Wissen transferiert. ${ }^{16}$ Die Konzentrierung auf die Termini korrespondiert überdies mit der Ansicht von Möhn und Pelka, die meinen, dass „Lexik das deutlichste Merkmal ist, durch welches sich die Fachsprache von der Allgemeinsprache unterscheidet. ${ }^{\text {"17 }}$ Diesen Annahmen folgend, soll das besondere Augenmerk auf die hochfrequenten Nominalisierungsstrategien im untersuchten Bereich gerichtet werden. Dieses Verfahren soll die wichtigsten Eigenschaften der beschriebenen

${ }^{15}$ PC 20 (Kontrolle, Budgetierung), OD 20 Büroorganisation und Kommunikation / BT 10 ( Eisenbahnbau/ Bauplanung), AT 80 (Neue, andere Verkehrssysteme), BT 44 - Weichen und Kreuzungen (Weichen do quantitative Aspekte), MA 10 (Verkehrswesen - Transport) - MT 40 (Fahrzeug, Eisenbahntriebfahrzeug, allgemeine Termini), MT 42 (Hauptgüterbahnhof), MT 45 (Dampftriebfahrzeug) MT 50 (Wagen allgemeine Termini), MT 52 (Wagen/Personenverkehr) MT 54 (Wagen: Güterverkehr) PR 10 (Bahnanlagen), PR 20 (Zugfahrt) PR 30 (Rangierdienst) PR 35 (Zugförderungsdienst) PR 40 Betriebsführung und Überwachung, PR 50 - Fahrplanwesen / bunt gereihte Sammelwagengruppe, NT 70 (Streckentechnik)

${ }^{16}$ Vgl. Fraas, Claudia: Lexikalisch-semantische Eigenschaften von Fachsprachen. In: Hoffmann, Lothar/Kalverkämper, Hartwig / Wiegand, Herbert Ernst (Hgg.): Fachsprachen / Languages for Special Purposes. - Ein internationales Handbuch zur Fachsprachenforschung und Terminologiewissenschaft. Berlin, New York 1998, S. 428-438.

${ }^{17}$ Möhn Dieter/ Pelka, Roland: Fachsprachen. Eine Einführung. Tübingen 1984, S. 14. 
Bahnterminologie sichtbar machen und ferner auch Rückschlüsse auf Spezifik der Sprachsysteme erlauben, innerhalb deren diese Fachbegriffe gebildet werden.

\section{Zu den hochfrequenten Wortbildungsprodukten}

Während der Auswertung des Untersuchungskorpus wurde die eindeutige Dominanz der Komposita registriert. ${ }^{18}$ Die zahlenmäßig stärkste Präsenz der Zusammensetzungen nimmt nicht wunder, da die substantivische Komposition im Deutschen als der produktivste Wortbildungsmechanismus gilt, dessen Bildung praktisch uneingeschränkt erfolgt. ${ }^{19}$

\subsection{Zwei und dreigliedrige NN-Komposita}

Innerhalb der Zusammensetzungen überwiegen zwei- und dreigliedrige substantivische Determinativkomposita. Im Hinblick auf die Bedeutungsrelationen unter den Konstituenten lässt sich bei diesen zusammengesetzten Nominationseinheiten in den meisten Fällen eine Finalbeziehung ${ }^{20}$ erkennen. Die bestimmungsorientierte Semantik zwischen den Wurzeln der N-NKomposita drückt sich im polnischen Fachwortschatz des Eisenbahnwesens primär durch die präpositionalen Wortgruppen aus: Betonzug (pociąg do układania betonu), Fischzug (pociąg do przewozu ryb), Schutterzug (pociąg do przewozu thucznia), Güterhalle (hala do składowania towarów), Berufszug (pociąg do przewozów pracowniczych). Bei den so aufgebauten deutschen Komposita wird die Prozess-Relation, welche die Teilbedeutungen des Determinans und Determinatums verbindet, als redundant ausgeblendet, was man an der Bedeutungsparaphrase

$1873 \%$ aller Lexeme

${ }^{19}$ Vgl. Lohde, Michael: Wortbildung des modernen Deutschen. Ein Lehrund Übungsbuch. Tübingen: 2006, S. 63.

${ }^{20} \mathrm{Vgl}$. Fleischer, Wolfgang/ Barz, Irmhild: Wortbildung der deutschen Gegenwartssprache. Tübingen: 2007, S. 98-103. 
deutlich erkennen kann - ein Fischzug ist nämlich ein Zug, der für die Beförderung [S.M.] der Fische bestimmt ist. Bei den polnischen Übersetzungspaaren wird diese ausgesparte Bedeutungskomponente dagegen transparent, indem sie durch entsprechend deklinierte, polnische deverbale Ableitungen: składowanie (dt. Aufbewahrung), przewożenie (dt. Beförderung), kładzenie (dt. Legung) expliziert wird. Demselben semantischen Muster folgt die nächste eruierte Subgruppe der mehrelementigen Nomenkomposita, wobei diesmal der funktionelle Zweck durch die -ung-Derivate als Bestimmungsglieder exponiert wird. Die semantische Exponierung vollzieht sich durch die Vererbung der prozessualen Sememe der zugrundeliegenden Verbbasis an die links stehenden Suffigierungen. Diese Wirkung entfalten folgende Eisenbahn-Lexeme, wie Entkupplungsgabel (drążek do rozsprzęgania), Besandungsgleis (tor do zaopatrywania w piasek), Desinfizierungsgleise (tory do odkażania), Nachbeeisungsbahnhof (stacja do zaopatrywania wagonów-chłodni w lód), Fahrleitungsbeobachtungsfahrzeug (pojazd do obserwacji sieci trakcyjnej), Reinigungsgleise (tory do mycia). Die ererbte Handlungsorientiertheit ${ }^{21}$ der derivativen Bestimmungsglieder wird im Polnischen durch die präpositionale Nominalphrase realisiert.

Die Finalbeziehung, die die innere Wortbildungsbedeutung der meisten kompositionellen Benennungen der deutschen Bahnsprache prägt, wird im Polnischen darüber hinaus durch ein Substantiv mit nachgestelltem Adjektivattribut ausgedrückt, z. B. Gleisbremse (hamulec torowy), Schlafwagen (wagon sypialny), der Güterwagen (wagon towarowy), Schutzweiche (rozjazd zabezpieczający), die Bergbahn (kolej górska), das Zufahrtgleis (tor dojazdowy), Gleisanschlussentgelt (opłata bocznicowa), der Ausflugszug (pociąg wycieczkowy).

${ }^{21}$ Diese ist eine Konsequenz der Transitivität der Verbbasis der genannten Ableitungen. 


\subsection{Rektionskomposita und Zusammenbildungen}

Die zweitstärkste Subgruppe bilden Rektionskomposita mit Agens-Verhältnis zwischen den Konstituenten. Im Folgenden sollen als Rektionskomposita solche Zusammensetzungen eingestuft werden, bei denen das Bestimmungswort gegenüber dem Grundwort im analogen Verhältnis wie eine Ergänzung zum Valenzträger steht. ${ }^{22}$ Erwartungsgemäß dominieren in dieser Gruppe die Zusammensetzungen mit Nomen Agentis (Akteur) - also deverbaler Zweitkonstituente, die von einem transitiven Verb abgeleitet wurde. Mit Hilfe der auf die Weise gebildeten Rektionskomposita wird in erster Linie die Personalstruktur der Bahn nachgezeichnet. Trotz der Tatsache, dass fast alle Benennungen für Bahnbedienstete eine verbale Gruppe als Basis haben, kann man innerhalb dieser Klasse gewisse Unterschiede konstatieren. Die größte Vorkommensquote belegen dabei verbbasierte Rektionskomposita, denen im Polnischen die Nominalgruppen gegenüberstehen. Bei den Stellenbezeichnungen, wie: Fahrdienstleiter (dyżurny ruchu), Betriebsleiter (naczelnik służby eksploatacyjnej), Wagenverteiler (dyspozytor wagonów), Weichenräumer (czyśćiciel rozjazdów), Gleisbremsenbediener (ustawiacz hamulców torowych), Streckenlokführer (maszynista lokomotyw linowych) wird der zugrundeliegende Struktural-Kasus, im gegebenen Fall - Akkusativ, durch das polnische Genitivattribut wiedergegeben. Die polnischen Äquivalente decken sich demnach in den meisten Fällen mit der Bedeutungsparaphrase der deutschen Termini, z. B. Wagenverteiler Verteiler der Wagen (pl. dyspozytor wagonów), der Fahrdienstleiter - der Leiter des Fahrdienstes (dyżurny ruchu) usw. Anders verhält es sich bei Rektionskomposita: Lokomotivheizer (pala$\mathrm{cz}$ parowozowy), Grenzspediteur (spedytor graniczny), Bahnhofsschaffner (bileter peronowy) und Bahnsteigschaffner

${ }^{22}$ Vgl. Vater, Heinz: Einführung in die Sprachwissenschaft. München 1994, S. 86-87. 
(dyżurny peronowy), deren Konstituente in einem quasi LokalVerhältnis (B vollzieht/ agiert in A) ${ }^{23}$ zueinanderstehen. Die Adjektivattribute der polnischen Fachbegriffe gelten dabei als Produkte der Adjektivierung von Substantiven, die im Deutschen die angedeuteten Einsatzbereiche bzw. Kompetenzbereiche benennen, z.B. granica - graniczny bei spedytor graniczny (dt. Grenzspediteur). Bei den Begriffen für Eisenbahnbedienstete lassen sich außerdem einige Beispiele für Zusammenbildung ausfindig machen, die als Sonderfall des Kompositums gelten, ohne die formalen Vorgaben dieses einzuhalten. Die Zusammenbildung beinhaltet nämlich ein Element, das weder als Konfix noch als ein Wort frei vorkommt. ${ }^{24} \mathrm{Zu}$ den in RailLexic enthaltenen Zusammenbildungen, die, nebenbei bemerkt, wegen ihrer scheinbaren Ähnlichkeit mit den obigen Rektionskomposita oftmals verwechselt werden, gehören solche Belege wie: Gepäckträger (bagażowy), Rangierarbeiter (manewrowy), Weichensteller (zwrotniczy), Hemmschuhleger (płozowy), denen im Polnischen implizite Derivate (substantivierte Adjektive) entsprechen. Den Rektionskomposita und Zusammenbildungen begegnet man innerhalb des analysierten Bahnwortschatzes nicht nur bei den Nomina Agentis, sondern auch bei den Nomina Instrumenti in Form der Bahngerätschaften, wobei diese Begriffe semantisch teil-motiviert sind, da sie auf ein Gerät referieren, das in der Wortstruktur nicht explizit genannt ist. Bei den dazugehörigen Rektionskomposita: Ölförderer (podajnik oleju), Leistungsschalter (wyłącznik mocy), Achszähler (licznik osi), Fahrstraßenspeicher (rejestrator dróg przebiegu), Weichenzungenprüfer, Zungenprüfer (urządzenie kontroli położenia iglic), Wagenbeschleuniger (przyspieszacz wagonów) und den Zusammenbildungen: Schichtdiensteinteiler (wykres pracy

${ }^{23}$ Eine solche Bedeutungsrelation soll im Folgenden als Ergänzung der Wortbildungsbedeutung „Lokal“" bei Fleischer/Barz (1995:111) betrachtet werden.

${ }^{24}$ Vgl. Engel, Ulrich: Deutsche Grammatik. Heidelberg 1988, S. 522. 
turnusowej), Fahrtrichtungsumsteller (nastawnica zmiany kierunku jazdy), Gegenausgleichträger (wieszak przeciwwagi), Wagenwascher (myjnia wagonów pasażerskich), Bodenentlader (wagon z odchylną podłogą) sieht man, dass die polnischen Entsprechungen fast ausschließlich auf Nominalgruppen mit einem Genitivattribut basieren, wo die eindeutige Bedeutungsstruktur der deverbalen Substantive, mit denen die Wortgruppen eingeleitet werden, die richtige Enkodierung erleichtert. Relativ zahlreich vertreten sind unter den Rektionskomposita der Bahnsprache ebenfalls kompositionelle Nomina Actionis (Vorgänge), deren Grundwörter explizite Substantivderivate auf -ung sind, z. B. Frachtpreisberichtigung (korekta opłaty przewozowej), Fahrplanordnung (układanie rozkładu jazdy), Fahrwegausrüstung (wyposażenie toru jezdnego), Fahrwegsicherung (zabezpieczenie drogi przewozu), Fahrbahndurchbiegung (ugięcie toru jezdnego), Zugüberholung (wyprzedzenie pociągu), Kesselspeisewasseraufbereitung (zmiękczenie wody kotłowej). Wie alle vorigen Rektionskomposita werden auch diese Wortbildungskonstruktionen durch den Inkorporierungsprozess gekennzeichnet, der in der Übertragung des vom Verb ererbten Arguments in die semantische Struktur des Grundwortes besteht. ${ }^{25}$ Am Beispiel des Begriffes „Frachtpreisberichtigung“ kann dies so erklärt werden, dass das verbbasierte Determinatum, „Berichtigung“ vom Objektargument „Frachtpreis“" semantisch bestimmt wird. Bezeichnenderweise werden die rechtsstehenden valenzgebenden Deverbativa bei der Übersetzung ins Polnische meistens durch prozessuale Verbalsubstantive mit dem Formativ -nie und -cie realisiert, die von den Genitivattributen mit anschließenden Adjektiven begleitet werden.

In der Gruppe der zwei- und mehrgliedrigen Zusammensetzungen des Grundtyps Substantiv + Substantiv registrierte

${ }^{25}$ Welke, Klaus: Wortbildung und Valenz. In: Giessen, Hans/ Lenk, Hartmut (Hgg.): Der Ginkgo-Baum. Germanistisches Jahrbuch für Nordeuropa. 13. Folge. Helsinki 1995, S. 228-236. 
man überdies Benennungen, die durch das Possessivverhältnis zwischen den Konstituenten semantisch gekennzeichnet sind und im Polnischen durch genitivische Gruppen ausgedrückt werden, z. B. Wagonklasse (klasa wagonu), Abstellgleisgruppe (grupa torów odstawczych), Haltegenauigkeit (dokładność zatrzymania), Lokomotivpark (park lokomotyw), Trassenkorridor (korytarz trasy), Lokomotivbestand (ilostan lokomotyw), Ablaufgleis (tor staczania). Die überdurchschnittliche Produktivität der Adjektivattribute und der Genitivgruppen, die den bisher angeführten Belegen zu entnehmen war, liegt hauptsächlich daran, dass das Polnische bei der semantischen Eingrenzung in der Regel zum nachgestellten Adjektiv tendiert, wohingegen dieselbe Wirkung im Deutschen in den meisten Fällen durch das Determinans erzielt wird. ${ }^{26}$

\subsection{Polymorphische Substantivkomposita}

Als separate Gruppe innerhalb der extrahierten Zusammensetzungen wurden in meiner Untersuchung die polymorphischen Komposita behandelt. Als solche sind im Folgenden nach Fleischer/Barz Wortzusammensetzungen zu betrachten, die aus mindestens vier und mehr Grundmorphemen bestehen. ${ }^{27}$ Trotz ihres ausgebauten Formativs fanden die mehrfach zusammengesetzten Termini reichlich Eingang in die Terminologie der Eisenbahner $^{28}$, was sich mit der Annahme erklären lässt, dass sie in der Regel semantisch motiviert sein müssen, um trotz der hohen Informationsdichte im Kommunikationsprozess reproduziert werden zu können und folglich die Schwelle der okkasionellen Ausdrücke zu überwinden. Diese Sichtweise mag einer weit verbreiteten Behauptung zuwiderlaufen, die besagt,

${ }^{26}$ Vgl. Jeziorski, Jan: Substantivische Nominalkomposita des Deutschen und ihre polnischen Entsprechungen. Wrocław 1983, S. 72.

${ }^{27} \mathrm{Vgl}$. Fleischer, Wolfgang/ Barz, Irmhild: Wortbildung der deutschen Gegenwartssprache. Tübingen: 2007, S. 97.

${ }^{28} 14$ Prozent aller aufgenommenen Komposita [S.M.]. 
dass der semantische Inhalt jener Bandwurmkomposita erst durch Zugriff auf ihren Verwendungskontext und Aktivierung des Allgemeinwissens des Rezipienten eindeutig erschlossen werden kann. ${ }^{29}$ Die von mir gewagte These findet allerdings ihre Begründung in der Spezifik der beschriebenen Terminologie-Datenbank, da, wie schon zuvor angedeutet, alle RailLexicBegriffe von den UIC-Terminologen zunächst anerkannt werden mussten, was das Risiko des Eindringens von lexikalischen Eintagsfliegen und schwerverständlichen Bandwurmlexemen verringern mochte. Die überwiegende Mehrheit ${ }^{30}$ der in RailLexic kodifizierten Mehrfachkomposita besteht nur aus Substantiven. Die mit Abstand größte Gruppe bilden dabei Begriffe für bewegliche Verkehrsträger und technische Anlagen der Bahninfrastruktur, deren polnische Pendants die Form der Präpositionalattribute mit Finalbedeutung haben, z. B.: Fährbootreisezugwagen (wagon osobowy do przewozu promem), Fahrleitungsbeobachtungswagen (wagon osobowy do przewozu promem), Kraftfahrzeugverladelampe (rampa do załadunku samochodów), Fahrleitungsprüfwagen (wagon do inspekcji sieci trakcyjnej), Güterwagenentlademaschine (urządzenie do rozładunku wagonów), Kesselsteinbeseitigungsgerät (urządzenie do usuwania kamienia kotłowego), Vororttriebwageneinheit (zespół trakcyjny do obsługi ruchu podmiejskiego), Schotterselbstentladewagen (wagon samowyładowczy do przewozu podsypki), Warmblocktransportwagen (wagon do przewozu gorących wlewków), Bahnsteigkartenautomat (automat do biletów peronowych), Autotransportwagen (wagon do przewozu samochodów). Kontrastiv betrachtet, kann die polnische Präposition $d o$, mit deren Hilfe der direkte Bezug auf die Funktion bzw. Einsatzweise der benannten Gegenstände im Polnischen genommen wird, für ein Äquivalent der symbolischen Grenze zwischen dem

${ }^{29}$ Vgl. Donalies, Elke: Die Wortbildung des Deutschen. Ein Überblick. Tübingen 2002, S. 65.

${ }^{30}$ Fast sämtliche Mehrfachkomposita werden so gebildet [S.M.] 
Bestimmungs- und Grundwort der deutschen Komposita interpretiert werden.

\subsection{Zusammensetzungen mit Verbstamm als Erstglied}

Die durchgeführteAnalyse ergab, dass die Zusammensetzungen, die in ihrer Struktur andere Wortarten als Nomen aufweisen, eher als eine Randerscheinung der untersuchten Terminologie anzusehen sind. ${ }^{31}$ Als einziger produktiver Typ erwiesen sich in diesem Zusammenhang Termini mit Verbstamm als Erstglied, die in ihrem semantischen Spektrum auf Zug- bzw. Wagenbewegungen referieren. Bezeichnenderweise dominieren bei diesen Nominaleinheiten die Patiens-Komposita ${ }^{32}$ (mit B wird A getan) mit präfigiertem Verbstamm als Erstglied, wie z. B. Abstellanlage (tory odstawcze), Abfallberg (górka rozrządowa), Ablaufbremse (hamulec torowy), Einfahrgruppe (grupa przyjazdowa), Überfahrgleis (tor przejazdowy), Umfahrgleis (tor objazdowy), Umladewagen (wagon przeładunkowy), Ladelänge (długość rozładunkowa), denen in der polnischen Terminologiedatenbank Nominalphrasen aus einem Nomen und nachgestelltem Adjektivattribut entgegenstehen.

\section{Derivation durch deverbative Ableitungen}

Unter den Produkten der Derivation, deren Vorkommensquote im Untersuchungskorpus bei $11 \%$ liegt, überwiegen die deverbativen Ableitungen mit dem Suffix -ung, die als Prozessnamen verwendet werden, z. B.: Zerlegung (rozdzielanie składu), Abzweigung (rozwidlenie lini), Stilllegung (zamknięcie lini dla przewozów), Unterhaltung (utrzymanie), Bedeckung (przykrycie ładunku), Bereitstellung (podstawienie pociągu), Auskupplung (rozłączanie sprzęgu), Überpufferung (zderzenie się

${ }^{31}$ Nur 3\% aller Komposita-Belege.

${ }^{32}$ Vgl. Fleischer, Wolfgang/ Barz, Irmhild: Wortbildung der deutschen Gegenwartssprache. Tübingen: 2007, S. 112. 
zderzaków). Die zitierten deutschen Derivate können als Beispiele für Terminologisierung ${ }^{33}$ fungieren, da sie aus dem gemeinsprachlichen Wortschatz genommen und in den Fachwortschatz der Eisenbahn transferiert wurden. Während dieses Transfers erfuhr der semantische Gehalt dieser Derivate wesentliche Erweiterung, was allerdings erst bei der Gegenüberstellung mit ihren polnischen Pendants deutlich wird. Das konstruktionsexterne Bedeutungselement - in diesem Fall ein Objekt -, welches der Wirkung der benannten Prozesse unterzogen wird, wird im Polnischen konsequent durch ein Genitivattribut offengelegt. Die besondere Effizienz der deverbalen Konvertate auf -ung in der Fachsprache des Schienenverkehrs kann als klarer Beweis für Gültigkeit der These Eisenbergs gelten, wonach die expliziten Derivate mit dem Suffix -ung einen Anteil von etwa $80 \%$ aufweisen und somit die produktivste Ableitungsform des Deutschen sind. ${ }^{34}$

\subsection{Konversion am Beispiel der substantivierten Infinitive}

Als eine Sonderart der Derivation soll an dieser Stelle ferner auf die Konversion eingegangen werden. Zu den Produkten der Konversion, die man als Übertritt in eine andere Wortart ohne Zuhilfenahme der zusätzlichen Wortbildungselemente definiert ${ }^{35}$, gehören etwa 4\% der Fachbegriffe in den untersuchten Domänen. Die höchste Frequenz kann in dieser Hinsicht bei den substantivierten Infinitiven der Präfixverben belegt werden, z. B.: Abkuppeln (rozprzęganie), Ablaufen (dobieganie), Absetzen (osadzanie), Auffahren (najeżdżanie), Bespannen (doczepianie lokomotywy do pociągu), Beistellen (doczepianie

${ }^{33}$ Vgl. Drewer, Petra/ Schmitz, Klaus-Dirk: Terminologiemanagement. Berlin: 2017, S. 37-39.

${ }^{34}$ Vgl. Eisenberg, Peter: Grundriß der deutschen Grammatik. Bd. 1. Das Wort. Stuttgart: 1998, S. 266.

${ }^{35}$ Vgl. Wolfgang Fleischer, Irmhild Barz, Wortbildung der deutschen Gegenwartssprache. Tübingen 1995, S. 209-211. 
wagonu), Zurücksetzen (cofanie składu manwerowego). Fast allen der bereits angeführten Infinitivkonvertate ist der gemeinsame Referenzbereich anzuerkennen, da sie sich thematisch auf die Rangiermanöver ${ }^{36}$ beziehen und so den Benennungsbedarf auf diesem Feld abdecken. Die prozessuale Referenz der substantivierten Infinitive lässt sich auf ihr charakteristisches Merkmal zurückführen, und zwar auf die Fähigkeit, das benannte Geschehen in seiner ganzen Dauer zu erfassen, die ihnen u.a. von Fleischer und Barz attestiert wurde. ${ }^{37}$ Auffällig bei den dargelegten Lexemen ist die einheitliche Benennungsform ihrer polnischen Gegenpaare, welche, in Anbetracht des Mangels an substantivierten Infinitiven im Polnischen, die bereits erwähnte durative Bedeutung durch deverbale Ableitungen aktivieren.

\section{Resümee}

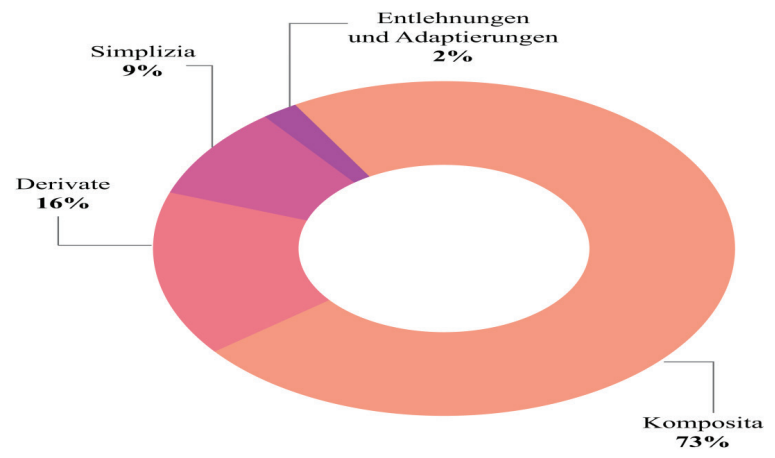

(Bild 1 - Wortbildungsmechanismen in der Eisenbahnterminologie [S.M.])

${ }^{36}$ Als Rangieren soll hier nach DB Fahrdienstvorschrift 408 das Bewegen von Fahrzeugen verstanden werden.

${ }^{37}$ Vgl. Wolfgang Fleischer, Irmhild Barz, Wortbildung der deutschen Gegenwartssprache. Tübingen 1995, S. 210. 
Bei der Schilderung der gewonnenen Erkenntnisse richtete ich mich nach dem methodologischen Ratschlag Hoffmanns, der als ein zuverlässiges Deskriptionsverfahren der Fachsprachenforschung den Produktivitätsvergleich empfiehlt. ${ }^{38}$ Die Angabe der höchstfrequenten Wortbildungsmechanismen gibt Aufschluss über das Sprachsystem, innerhalb dessen die jeweilige Fachterminologie konstituiert wird. Ausgehend von dieser Prämisse wurde in dem deutschen Fachwortschatz des Eisenbahnwesens eine dominante Neigung zu den kompositionellen Nominalisierungen konstatiert, was in der breiteren Perspektive mit der These Roelckes übereinstimmt, der zufolge die fachsprachlichen Register eine noch stärkere Tendenz zur synthetischen FormalsdieGemeinspracheoffenbaren. ${ }^{39}$ DieSyntheseprägung ${ }^{40}$ wird im deutschen Eisenbahnwortschatz vor allem von den Komposita gewährt. Die meisten dieser kompositionellen Einheiten sind Nomina Agentis, Nomina Instrumenti und Nomina Actionis, weshalb man schlussfolgern kann, dass die Eisenbahnsprache vordergründig handlungs- und zielorientiert ist. Die Analyse der polnischen Äquivalente ergab den höchsten Prozentsatz der genitivischen Gruppen und Nominalphrasen mit einem Adjektivattribut, was wiederum den analytischen Charakter des Polnischen widerspiegelt. Die dargelegten Belege mögen schließlich als Abbild der identitätsstiftenden Rolle der deutschen Eisenbahn gesehen werden, da, wie aus den quantitativen Ergebnissen hervorgeht, etwa 97\% aller Eisenbahnfachwörter auf der Basis des indigenen Sprachmaterials gebildet werden. Die im Rahmen dieses Vortrags unberücksichtigt gebliebenen Entlehnungen stellen in Bezug auf das Thema der

${ }^{38} \mathrm{Vgl}$. Hoffman, Lothar: Syntaktische und morphologische Eigenschaften von Fachsprachen. In: Hoffmann, Lothar/Kalverkämper, Hartwig / Wiegand, Herbert Ernst (Hgg.): Fachsprachen / Languages for Special Purposes. -Eininternationales Handbuch zur Fachsprachenforschung und Terminologiewissenschaft. Berlin, New York 1998, S. 89-93.

${ }^{39}$ Vgl. Roelcke, Thorsten: Fachsprachen. Berlin: 2005, S. 77-79. ${ }^{40}$ Ebd. 
morphologischen Nominationsstrategien eine marginale Erscheinung dar, da sie entweder als diastratisch begrenzte Simplizia ${ }^{41}$ oder als Elemente von längeren Synonymreihen auftreten. Die überwältigende Mehrheit der heimischen Wortbildungsprodukte in RailLexic kann schließlich symbolisch als eine Chance gesehen werden, dass die eingangs skizzierte Bindung zwischen der deutschen Identität und der Eisenbahn in der Sprache erhalten bleibt.

\section{Literaturverzeichnis}

Donalies, Elke: Die Wortbildung des Deutschen. Ein Überblick. Tübingen: Günter Narr Verlag 2002.

Drewer,Petra/Schmitz,Klaus-Dirk:Terminologiemanagement. Berlin 2: Springer Vieweg 2017.

Ebeling, Klaus: Die Fortentwicklung des Europäischen Eisenbahnsystems - ,Interoperabilität ${ }^{`}$ auch auf sprachlicher Ebene. In: Gerstenkorn, Alfred/ Hums, Lothar/ Schmidt, Arnold (Hgg.): Die Sprache der Bahn. Zur deutschen Eisenbahnsprache im europäischen Kontext. Frankfurt a. M.: DGI 2006, S. 291-303.

Eckermann, Johann Peter: Gespräche mit Goethe in den letzten Jahren seines Lebens. Wiesbaden: Brockhaus 1975. Eisenberg, Peter: Grundriß der deutschen Grammatik. Bd. 1 . Das Wort. Stuttgart: Metzler 1998.

Engel, Ulrich: Deutsche Grammatik. Heidelberg: Groos 1988. Fleischer, Wolfgang/ Barz, Irmhild: Wortbildung der deutschen Gegenwartssprache. Tübingen: Max Niemeyer Verlag 2007.

Fraas, Claudia: Lexikalisch-semantische Eigenschaften von Fachsprachen. In: Hoffmann, Lothar/ Kalverkämper, Hartwig / Wiegand, Herbert Ernst (Hgg.): Fachsprachen / Languages for Special Purposes - Ein internationales

${ }^{41}$ Z. B.: Billet, Coupé, Perron, Kondukteur - als Begriffe, die nur in der Schweiz gebräuchlich sind. 
Handbuch zur Fachsprachenforschung und Terminologiewissenschaft. Berlin, New York: Walter de Gruyter 1998, S. 428-438.

Gall, Lothar/ Pohl, Manfred: Die Eisenbahn in Deutschland von den Anfängen bis zur Gegenwart. München: C. H. Beck 1998.

Heine, Heinrich: Sämtliche Schriften in zwölf Bänden. Berlin: hrsg. v. Klaus Briegleb 1981, S. 449.

Hoffman, Lothar: Syntaktische und morphologische Eigenschaften von Fachsprachen. In: Hoffmann, Lothar/ Kalverkämper, Hartwig / Wiegand, Herbert Ernst (Hgg.): Fachsprachen / Languages for Special Purposes - Ein internationales Handbuch zur Fachsprachenforschung und Terminologiewissenschaft. Berlin, New York: Walter de Gruyter 1998, S. 89-93.

Jeziorski, Jan: Substantivische Nominalkomposita des Deutschen und ihre polnischen Entsprechungen. Wrocław u.a.:Zakład Narodowy Imienia Ossolińskich, Wydawnictwo PAN 1983.

Lohde, Michael (2006): Wortbildung des modernen Deutschen. Ein Lehr- und Übungsbuch. Tübingen: Narr.

Möhn, Dieter/ Pelka, Roland: Fachsprachen. Eine Einführung. Tübingen: Max Niemeyer Verlag 1984.

Roelcke, Thorsten: Fachsprachen. Berlin: Erich Schmidt Verlag 2005.

Welke, Klaus (1995): Wortbildung und Valenz. In: Giessen,

Hans/ Lenk, Hartmut (Hgg.): Der Ginkgo-Baum.

Germanistisches Jahrbuch für Nordeuropa. 13. Folge.

Helsinki: Finn Lectura 1995, S. 228-236.

Vater, Heinz: Einführung in die Sprachwissenschaft. München:

Fink 1994, S. 86-87. 